\title{
Generalized Pareto Distributions-Application to Autofocus in Automated Microscopy
}

\author{
Reiner Lenz
}

\section{Linköping University Post Print}

\section{Tweet}

N.B.: When citing this work, cite the original article.

(C)2016 IEEE. Personal use of this material is permitted. However, permission to reprint/republish this material for advertising or promotional purposes or for creating new collective works for resale or redistribution to servers or lists, or to reuse any copyrighted component of this work in other works must be obtained from the IEEE.

Reiner Lenz , Generalized Pareto Distributions-Application to Autofocus in Automated Microscopy, 2016, IEEE Journal on Selected Topics in Signal Processing, (10), 1, 92.

http://dx.doi.org/10.1109/JSTSP.2015.2482949

Postprint available at: Linköping University Electronic Press

http://urn.kb.se/resolve?urn=urn:nbn:se:liu:diva-125679 


\title{
Generalized Pareto Distributions - Application to Autofocus in Automated Microscopy
}

\author{
Reiner Lenz
}

\begin{abstract}
Dihedral filters correspond to the Fourier transform of functions defined on square grids. For gray value images there are six pairs of dihedral edge-detector pairs on $5 \times 5$ windows. In low-level image statistics the Weibull- or the generalized extreme value distributions are often used as statistical distributions modeling such filter results. Since only points with high filter magnitudes are of interest we argue that the generalized Pareto distribution is a better choice. Practically this also leads to more efficient algorithms since only a fraction of the raw filter results have to analyzed. The generalized Pareto distributions with a fixed threshold form a Riemann manifold with the Fisher information matrix as a metric tensor. For the generalized Pareto distributions we compute the determinant of the inverse Fisher information matrix as a function of the shape and scale parameters and show that it is the product of a polynomial in the shape parameter and the squared scale parameter. We then show that this determinant defines a sharpness function that can be used in autofocus algorithms. We evaluate the properties of this sharpness function with the help of a benchmark database of microscopy images with known ground truth focus positions. We show that the method based on this sharpness function results in a focus estimation that is within the given ground truth interval for a vast majority of focal sequences. Cases where it fails are mainly sequences with very poor image quality and sequences with sharp structures in different layers. The analytical structure given by the Riemann geometry of the space of probability density functions can be used to construct more efficient autofocus methods than other methods based on empirical moments.
\end{abstract}

\section{INTRODUCTION}

In the following we will describe an autofocus method based on a sharpness function derived from basic facts from information geometry. We start with a description of a fast implementation of filter systems originating on the representation theory of the dihedral group $\mathrm{D}(4)$. This corresponds to the FFT implementation of the DFT in the case where the underlying group is the cyclic group (which is a subgroup of the dihedral group). The filter functions in these systems come naturally in pairs (and some single filters). The magnitude of such a two-dimensional vector is invariant under the group operations while the relation between the two filter results encode the group operation. For many image sources (including microscopy images) the combined magnitude values can be characterized with the help of the generalized extreme value distributions (GEV), of which the more commonly known Weibull distribution is one example (see for example [1] or [2] ). Using the GEVs to analyze the filter results requires that the contribution of flat regions with near-zero filter results has to be excluded in the GEV-fitting. This can be done using

R. Lenz is with the Department of Science and Technology, Linköping University, SE-60174 Norrköping, Sweden e-mail: reiner.lenz@liu.se. a threshold procedure where the selection of a good threshold is a non-trivial problem. The second problem of GEVbased methods is computational. More data means usually a better distribution fit but since the distribution fitting is an optimization procedure this also means that the cost of estimating the distribution parameters grows as the sample size becomes larger. In this paper we use a result from extremevalue statistics which shows that under certain conditions the tails of GEVs are generalized Pareto distributions (GPDs). This addresses both problems with the GEVs: the first step in the Pareto-based approach is to consider the distribution of the data exceeding a HIGH threshold. This means that near-zero filter results are automatically discarded and that the number of data points entering the Pareto-fitting is greatly reduced. The GPDs depend on three parameters where one of them (the location parameter) is the threshold used. Ignoring the location parameter (corresponding to moving the distribution to the origin) we find that the distributions are elements of a two-parameter manifold of probability distributions. On such a manifold we can introduce a metric structure with the help of the Fisher information matrix. On the resulting Riemannian manifold we can measure geometric properties like arc-length and curvature. We use this structure to introduce the determinant of the Fisher information matrix as a sharpness measure. Intuitively this is based on the idea that in blurred images the statistics of neighboring images in the focus sequence are very similar whereas a good focal position is a kind of critical point: the sharpness first increases and then decreases again with a maximum sharpness in the focal position. The sharpness measure based on the determinant of the Fisher information matrix tries to capture this behavior on the Riemannian manifold of the statistical distributions. In the last part of the paper we will investigate the performance of this method with the help of a benchmark dataset of microscopy images described in [3], [4]. We will show that for the focus sequences in the database the method results in a local maximum of the sharpness function in cases where the images contain sufficiently many meaningful objects points. Using a parametrized statistical model instead of methods based on empirical measurements, like means and variances, has the advantage that one can use the manifold structure of the distributions to construct more efficient search methods to find the focal position using a reduced number of image acquisitions. Methods from the theory of optimization on manifolds can be used to speed up the autofocus process as described in [1] but this is not included in the experiments described here. The main focus of this study is the investigation of the role of the GPDs in low-level signal processing. We illustrate its usefulness by constructing a sharpness function which is used in an 
autofocus application. The comparison of the proposed method with other autofocus methods and implementation details like the construction of optimal thresholds, fast estimations of the distribution parameters and construction of optimal focal sequences are outside the scope of this study (recent auto-focus related investigations are [5] and [6]).

\section{DihedRAL FILTERS}

Almost all digital images are functions defined on a square grid. The symmetry group of this grid is the dihedral group $\mathrm{D}(4)$ consisting of eight elements: four rotations and these rotations combined with one reflection on a symmetry axis of the square. The first step in low level image processing is often a linear filtering and results from the representation theory of $\mathrm{D}(4)$ show that filter functions given by the irreducible representations of the group $\mathrm{D}(4)$ are eigenfunctions of group-invariant correlation functions and thus principal components. They share the transformation properties of $\mathrm{D}(4)$ and they are computationally very efficient since they can be implemented using a reduced number of additions and subtractions only. The two-dimensional irreducible representation corresponds to a pair of gradient filters. In the following we will only use filter kernels of size $5 \times 5$ pixels. We divide the $5 \times 5$ window in so-called orbits which are the smallest $\mathrm{D}(4)$ invariant subsets. There is one orbit consisting of the center pixel, four orbits consisting of four points each (located on the axes and the corners of a square) and one orbit with the remaining eight points. This results in six pairs of filter results given by pairs of filter kernels of the form $\left(\begin{array}{ll}-1 & 1 \\ -1 & 1\end{array}\right)$ and $\left(\begin{array}{cc}-1 & -1 \\ 1 & 1\end{array}\right)$. The group theoretical structure of the $5 \times 5$ window is illustrated in Fig. 1 . It shows both, the coordinates of the pixels and the orbit (A-F) to which they belong. For the four point orbits B-E the filter pairs are just the ordinary $2 \times 2$ gradient filters. The eight point orbit contains two pairs of them, one computed from the four pixels at positions $(-1,-2),(2,1),(1,2),(-2,-1)$ and the other from the four pixels at positions $(1,-2),(2,-1),(-1,2),(-2,1)$. If $e_{1}$ and $e_{2}$ are the filter results computed with the help of the two filter kernels then it can be shown that the edgestrength $e_{1}^{2}+e_{2}^{2}$ is independent under all modifications of the underlying $5 \times 5$-window by elements of the dihedral group. For the $5 \times 5$ window we therefore obtain six edge-magnitude values and in the following we simply use their sum as the combined edge-strength in the window. More details can be found in [7], [8].

\section{Extreme VAlue AND PAREto Distributions}

For most pixels in an image the filter response to such a filter pair will be very small since neighboring pixels usually have similar intensity values and positions with large filter responses are most interesting. The statistical distribution of such edge-type filter systems has previously been investigated in the framework of the Weibull- or more generally in the framework of the generalized extreme value distributions (GEV) (see, for example [1], [9]-[13]). From the construction of the filter functions follows that the filter results follow a mixture distribution consisting of near-zero filter results and

\begin{tabular}{|c|c|c|c|c|}
\hline$(-2,-2) / \mathrm{E}$ & $(-1,-2) / \mathrm{F}$ & $(0,-2) / \mathrm{D}$ & $(1,-2) / \mathrm{F}$ & $(2,-2) / \mathrm{E}$ \\
\hline$(-2,-1) / \mathrm{F}$ & $(-1,-1) / \mathrm{C}$ & $(0,-1) / \mathrm{B}$ & $(1,-1) / \mathrm{C}$ & $(2,-1) / \mathrm{F}$ \\
\hline$(-2,0) / \mathrm{D}$ & $(-1,0) / \mathrm{B}$ & $(0,0) / \mathrm{A}$ & $(1,0) / \mathrm{B}$ & $(2,0) / \mathrm{D}$ \\
\hline$(-2,1) / \mathrm{F}$ & $(-1,1) / \mathrm{C}$ & $(0,1) / \mathrm{B}$ & $(1,1) / \mathrm{C}$ & $(2,1) / \mathrm{F}$ \\
\hline$(-2,2) / \mathrm{E}$ & $(-1,2) / \mathrm{F}$ & $(0,2) / \mathrm{D}$ & $(1,2) / \mathrm{F}$ & $(2,2) / \mathrm{E}$ \\
\hline
\end{tabular}

Fig. 1. Orbit structure of the window

the distribution of the significant edge magnitude values. This means that a threshold process is required before the extreme value distributions can be fitted. This can be avoided if we use the generalized Pareto distributions instead of the generalized extreme value distributions. The following selection of results from the theory of extreme value distributions may give a heuristical explanation why these distributions may be relevant in the current application.

The Three Types Theorem (originally formulated by Fisher and Tippett [14] and later proved by Gnedenko [15]) states the following: if we have an i.i.d. sequence of random variables $X_{1}, X_{2} \ldots$ and if $M_{n}=\max \left(X_{1}, X_{2} \ldots X_{n}\right)$ is the sample maximum then we find that if there exists (after a suitable renormalization) a non-degenerate limit distribution then this limit distribution must be of one of three different types. These types are known as the Gumbel-, Weibull- and Frechet distributions. One can combine these three distributions in a single generalized extreme value distribution (GEV). In the following we use the maximum likelihood estimators from the Matlab Statistical toolbox and we will therefore also use the definition and notations used there. The probability density function (pdf) of the GEV is defined as

$$
f(x ; k, \mu, \sigma)=\frac{1}{\sigma} \mathrm{e}^{-\left(1+k \frac{x-\mu}{\sigma}\right)^{-1 / k}}\left(1+k \frac{x-\mu}{\sigma}\right)^{-1-1 / k}
$$

where $\mu$ is the location, $\sigma$ is the scale- and $k$ is the shape parameter. In our implementation we don't consider the distributions with $k=0$ since we assume that in the case of real measured data we will encounter this case relatively seldom. Related to the GEV-distributions are the generalized Pareto distributions (GPD) with probability density functions defined as

$$
f(x ; k, \mu, \sigma, \theta)=\frac{1}{\sigma}\left(1+k \frac{x-\theta}{\sigma}\right)^{-1-1 / k}
$$

where $k$ and $\sigma$ are again the shape and scale and $\theta$ is the threshold parameter. For positive $k$ the support of the distribution is given by the half-axis $\theta<x<\infty$ and for negative $k$ by $\theta<x<-k / \sigma$. It is known that there is a close connection between the limit results for sample maxima and limit results for exceedances over thresholds. For a given probability distribution one can show that if the limit of the sample maxima converges to a GEV then the Peak-over-Threshold 


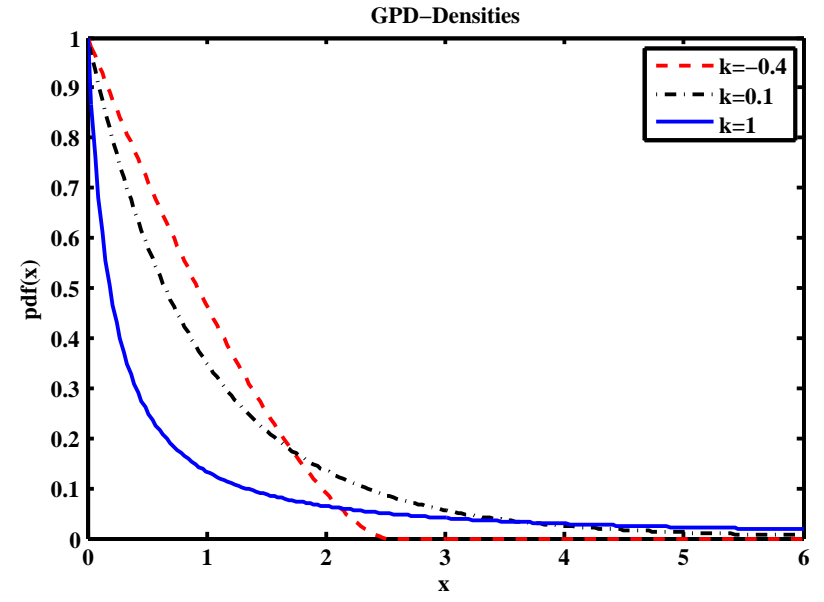

Fig. 2. Density functions of generalized Pareto distributions

limit converges to the GPD. In this case the shape parameters for both distributions are identical. For more details see [16] and Sec. 1.2 in [17]). The probability density functions of the three GPDs with parameters $\theta=0, \sigma=1, k=-0.4,0.1,4$ are shown in Figure 2.

Both the GEV and the GPD depend on three parameters. In our application we are only interested in the shape and the scale of the GPD since the location parameter is given by the threshold parameter. Therefore we consider the GPD as a class of distributions depending on the two parameters $k, \sigma$. For these distributions we can compute the $2 \times 2$ Fisher information matrix $\mathcal{G}(\eta)=\left(g_{k l}\right)$ with elements $g_{k l}$ and parameters $\eta_{1}=$ $k, \eta_{2}=\sigma$ defined as

$$
g_{k l}=\int_{x} \frac{\partial \log f(x ; \eta)}{\partial \eta_{k}} \frac{\partial \log f(x ; \eta)}{\partial \eta_{l}} f(x ; \eta) d x
$$

For the GPD we used Mathematica to obtain the following entries (note that $g_{22}$ is only defined for $k>-0.5$ ):

$$
\begin{aligned}
& g_{11}=\frac{2}{2 k^{2}+3 k+1} ; \\
& g_{12}=\frac{1}{2 k^{2} \sigma+3 k \sigma+\sigma} ; \\
& g_{22}=\frac{1}{(2 k+1) \sigma^{2}}
\end{aligned}
$$

We define the inverse of the determinant of the Fisher information matrix as sharpness function and we find the simple expression (see Figure 3 for a plot of the shape part $s(k)$ ):

$$
S(k, \sigma)=1 / \operatorname{det} \mathcal{G}(k, \sigma)=(k+1)^{2}(2 k+1) \sigma^{2}=s(k) \cdot \sigma^{2}
$$

We see that this sharpness function consist of two factors, the shape factor $s(k)$ and the squared scale factor $\sigma^{2}$. The scale factor corresponds to the often used variance-based sharpness functions but the new shape term $s(k)$ gives extra information about the reliability of the scale-estimate. For low values of $k$ the distribution is concentrated on finite intervals near zero, indicating a very weak edge content. For high values of $s(k)$ the tails become more significant and the sharpness estimate more reliable. In the current implementation we use the sum

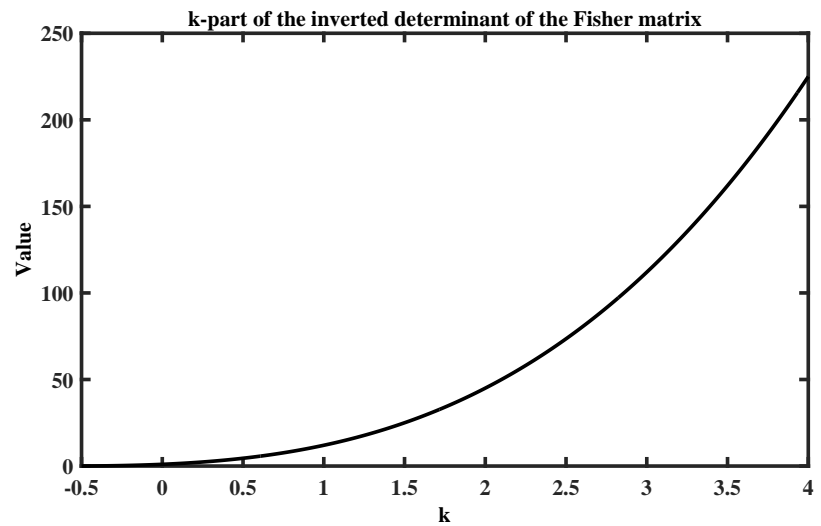

Fig. 3. Shape factor $s(k)$

of the six edge-magnitude filters as our scalar valued feature. All information about the scales and orientations contained in the full six-dimensional vector is thereby lost. It would therefore be interesting to generalize this model such that the full magnitude vector can be utilized. This can be done by using GPD's in higher dimensional data spaces as described in [18].

\section{DATABASE AND IMPLEMENTATION}

In our experiments we use the microscopy images from set BBBC006v1 in the Broad Bioimage Benchmark Collection which is described in [3], [4]. The images are available at http://www.broadinstitute.org/bbbc/BBBC006. The database contains 52224 images from 384 cells, measured at two positions and prepared with two different types of staining (we denote them as Hoechst and Phalloidin cells in the following). For each position and each cell a focus sequence consisting of 34 images was recorded. Each image consists of 696 x 520 pixels in 16-bit TIF format. The images show stained human cells and the imaging process is desribed as follows: For each site, the optimal focus was found using laser auto-focusing to find the well bottom. The automated microscope was then programmed to collect a $z$-stack of 32 image sets. Planes between $z=11$ - 23 are considered ground truth as in-focus images.

As mentioned above we first filtered an image with dihedral filters of size $5 \times 5$. Since these filters basically express the 25 -dimensional vectors in a new basis we get as a result a sequence of 25 filtered images. These filter results come in different types characterized by their transformation properties under the operations in $\mathrm{D}(4)$ (given by the irreducible representations of $\mathrm{D}(4)$ as explained above). Here we only consider those filter results that are invariant under all transformations (corresponding to the trivial representation, consisting of the sum of the pixel values on an orbit) and edge-type filter pair results (corresponding to the two-dimensional irreducible representation). A $5 \times 5$ grid consists of six orbits (with one, four and eight elements, see 1) and after the filtering we first select only those points for further processing where the value at the center pixel (one pixel orbit) is greater than the mean value of the pixels on the other orbits. In this way only positions with a local maximum intensity value are entering 
the statistical estimation. For these points we now select the twelve components of the filter results that transform like the two-dimensional representation of $\mathrm{D}(4)$ and we compute the length of this vector as a measure of the 'edge' strength in this point. In the group representation framework the length of this subvector should be computed as the usual Euclidean norm but we usually use the sum of the absolute values instead. This is faster and the results are comparable. For these length values we compute the Q-quantile and select all samples with a value greater than this quantile. A typical value for $\mathrm{Q}$ is 0.95 , so that about $5 \%$ of the selected local maxima points enter the GPD-fitting process. From the selected data we compute the minimum value $\theta$ and shift the data by subtracting $\theta$. Next we use the Matlab function gpfit which implements a maximumlikelihood estimator. For the first image in a focus sequence we use the standard startvalues in the optimization process and for the following images we use the result of the previous fitting as start values, thus reducing the execution time of the computationally intensive fitting process. The matlab-pseudo code is shown in Listing 1. For every focus sequence we selected the image with the maximum value of the inverse determinant as the detected optimal focal plane.

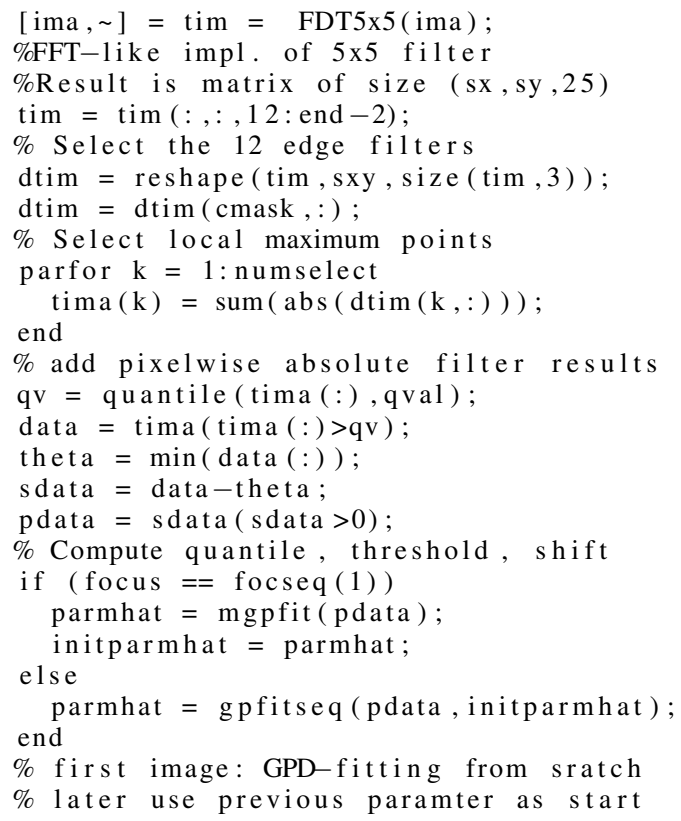

Listing 1. Matlab pseudo-code

\section{EXPERIMENTAL RESULTS}

In Figures 4, 5 and 6 we illustrate some of the relations between the GEVs and the GPDs. We use the filter results for one microscope image as an illustration. In Fig. 4 we see the distribution of the magnitude values over the 0.05 quantile threshold and the fitted GEV. We can clearly see the dominating influence of the low-level filter results. Increasing the threshold to the 0.75 quantile we can eliminate the influence of the low-level results, and in Fig. 5 we show the results of fitting a GEV and a GPD to the edge strength values. We see that both the GEV and the GPD give good fits but that the shape of the GPD is more similar to the empirical distribution.

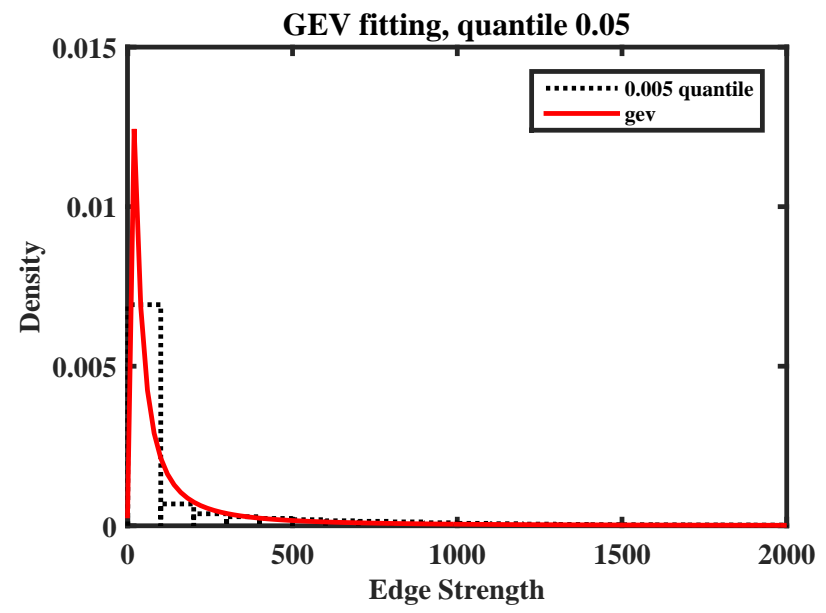

Fig. 4. Fitting generalized extreme value distributions to edge strength over the 0.05 quantile

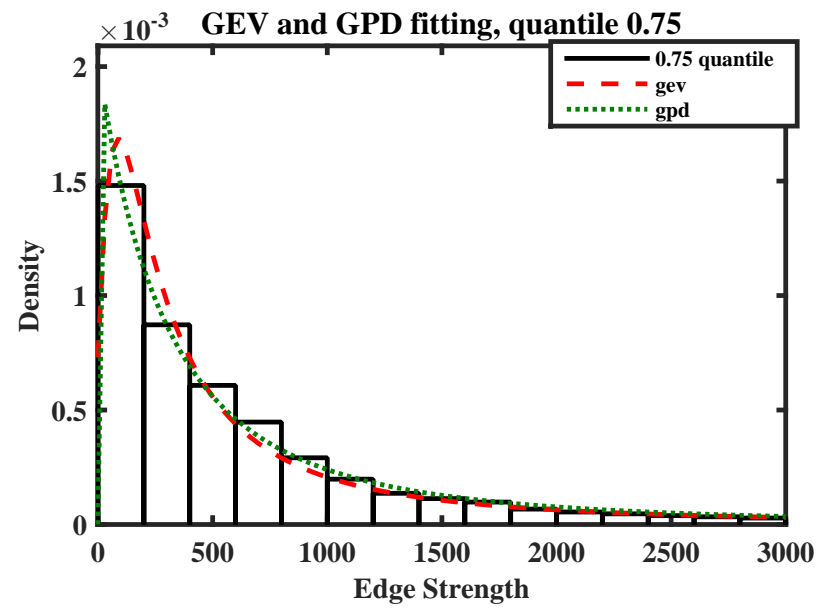

Fig. 5. Fitting generalized extreme value and generalized Pareto distributions to edge strength over the 0.75 quantile

The GEV distribution left of the maximum point has no real corresponding data. The result of an even higher threshold (now at the 0.95 quantile) is compared with the previous GPD fit in Fig 6. We can see that even at this high threshold the GPD fits the measured data as represented by the histograms (note that all distribution fittings involving GPDs are computed from the shifted data and therefore all distributions start from zero)

In the statistical part of the procedure described above there is only one parameter the user can choose: the value of the quantile based threshold parameter. For the experiments where we used the whole image as input a typical value we used is $\mathrm{Q}=0.95$. Other choices will be described later. We first analysed for how many sequences the detected focal plane lies in the range $z=11-23$ defined as the ground truth in the description of the database. There are $4 \cdot 384=1536$ sequences and in a typical case there were 23 sequences from cell Hoechst and 22 from Phalloidin where the focal plane estimate was found outside the ground truth region. The distributions of the detected focal positions are show in the histograms in Fig. 7. 


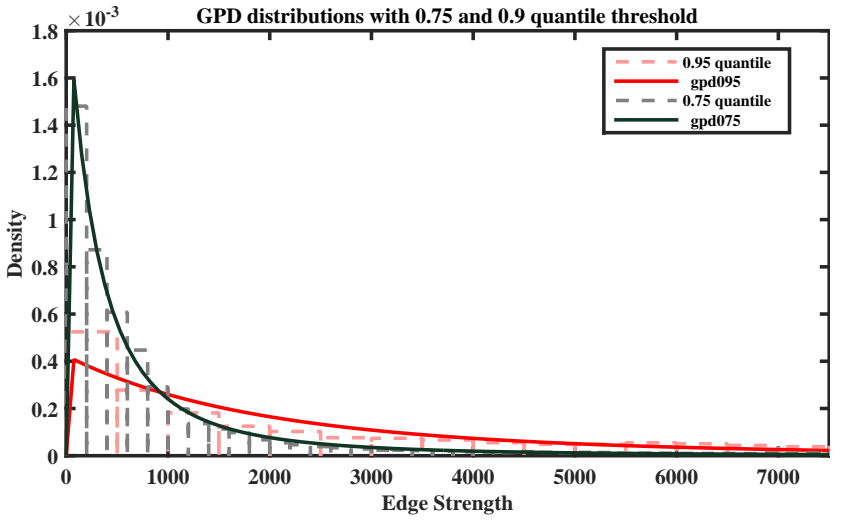

Fig. 6. Fitting generalized Pareto distributions to edge strength over 0.75 and 0.95 quantiles
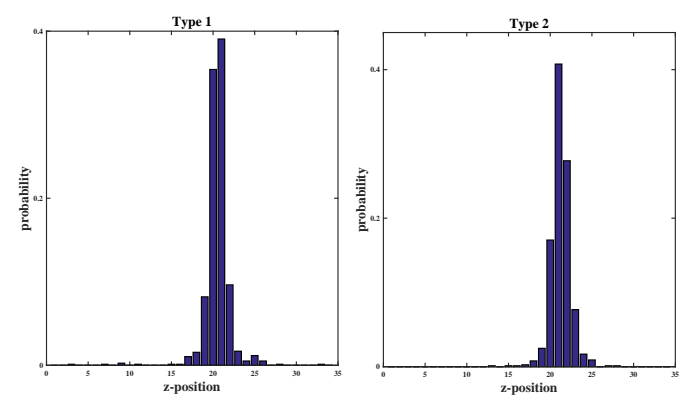

Fig. 7. Detected focus positions for 0.95 quantile threshold

The reasons for the failure to detect a focal position in the ground truth range are different for the two types, as we can see from the following, worst case, examples where we show the images in the focus stack and the corresponding sharpness function.

We start with cells of Hoechst where the estimated focal position is too small. A typical example of this type is shown in Fig. 8 where position 9 was selected as the sharpest image (the label of this specimen in the database is mcf-z-stacks-03212011_o13_s1_w1). In Fig 9 we see the corresponding sharpness function. From the slices we see that there are very few object points. As a result gpfit fitted a GPD for every image but the value of the shape parameter for some of the images (including those for position 19 and 21) was less than -0.49 which excluded these slices from the sharpness estimation. As a result image number 7 was selected as the sharpest image.

Next we select a sequence from a sequence of the same Hoechst but now with a focus position larger than the ground truth. From the sharpness function in Fig. 10 (label mcf-z-stacks-03212011_a03_s1_w1) we see that the sharpness function has indeed a second local maximum in the correct range but that the second maximum is larger. The reason for this behavior can be seen in the image sequence shown in Fig. 11. We see that also in this case very few object points can be found but we see also that a second object located in a different layer comes into focus and influences the sharpness estimates. For images of Phalloidin we do not find the error similar to the one shown in Fig 9 (label mcf-z-stacks-03212011_a01_s2_w2). The images have more structure and the wrong focal positions come all later in the sequence. An example where the estimated position is also 25 is shown in Figures 12 and 13. We see that the reason for the error is the same as before: very few object points and new object parts located in later layers.

Analyzing the cases where this global estimation process gives results outside the ground truth interval of slices 11 to 23 we can roughly distinguish the following types of errors. The first case consists of cases where the distribution fitting did not converge. This is typically the case for images with very little useful information, i.e. slices containing very few object points. In the second case the fitting was successful but the value of the estimated shape parameter was very low and the estimate is therefore not very reliable. Also in this case the number of useful object points was very small. In the third case we find multiple maxima of the sharpness function. Usually the first one lies in the ground truth interval but the second maximum comes later in the sequence. Visual inspection shows that this is often the case when high intensity cells lie in layers above or below the 'correct' focal plane. Such cases could be excluded when the search process stops after it has found the first significant maximum in the sharpness function. The value of the sharpness function gives also an indication of the reliabilty of the estimate as can be seen from the two sharpness functions in Fig. 14. The lower curve is the same as in Fig. 13 whereas the other curve is computed from a sequence, of the same cell Phalloidin, with a clear focus position in the required ground truth interval.

A common problem in the application of GPD-based methods is the selection of the high threshold. There are two conflicting aspects that have to be taken into account: On the one hand one would like to select as many data points as possible to get a good representation of the data. On the other hand one would like to have a high threshold to ensure that the remaining data really represents the tail of the distribution. In our experiments we used quantile-based thresholds only. The interested reader can find more information about the threshold selection in the relevant literature (see [19] for example). In the case where we used the 0.85 quantile threshold we found that 45 sequences (instead of the 23 in the 0.95 case) of the Hoechst images but only seven (instead of 22) of the Phalloidin image sequences resulted in falsely detected focal positions. In the current context the number of data points in the original distribution is clearly of importance here. In the experiments described so far we used information from the full $696 \times 520$ pixels image and we found that the 0.95 quantile threshold gave good results. In another sequence of experiments we selected $256 \times 256$ subimages from the center of each image. We then did the same filtering, thresholding and distribution fitting process as before. In these experiments we used the $0.75,0.8,0.85,0.9$ and 0.95 quantiles as thresholds. In these experiments we also started the computation with image number five in the stack and we computed the sharpness value for 25 images in the sequence only. We then computed for each of the sets how many focal positions where found in the ground truth interval. The results are summarized in 


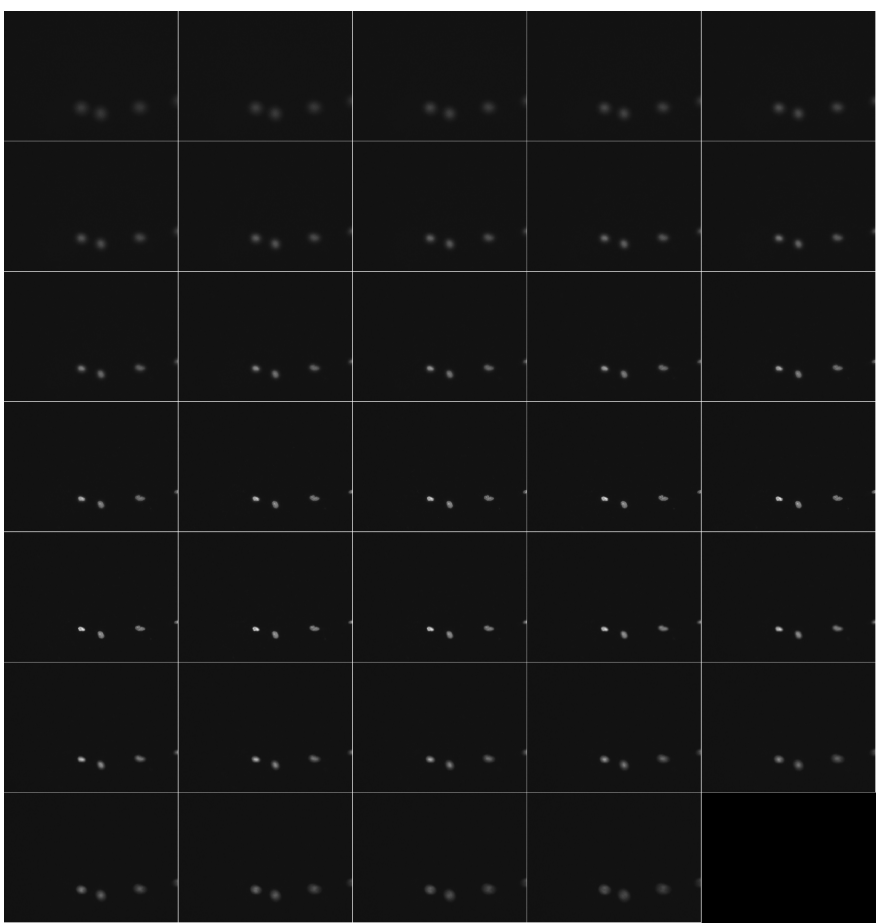

Fig. 8. Hoechst, detected focus position 9

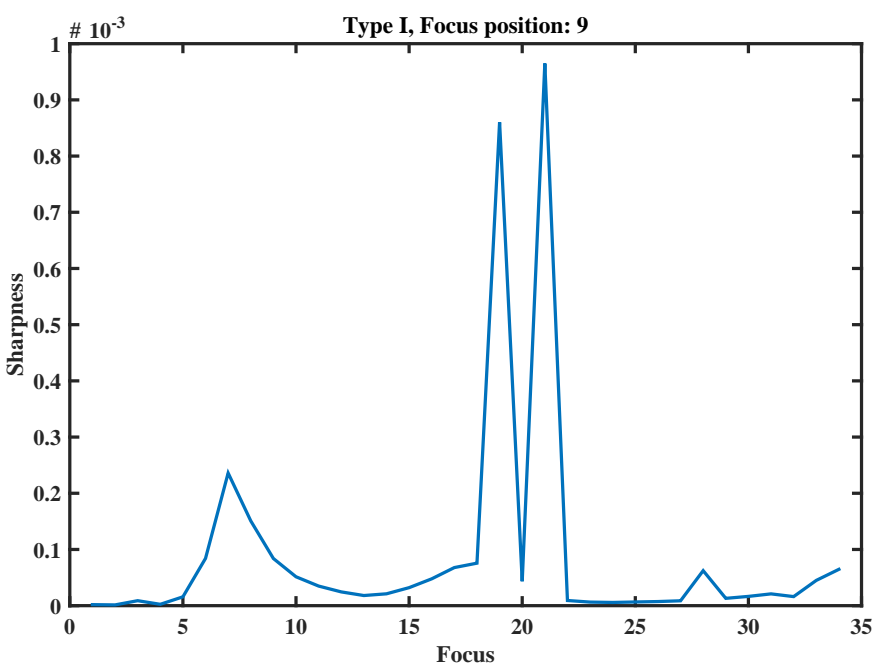

Fig. 9. Hoechst, detected focus position 9, sharpness function

Table I. We see that for both types of images the best results are obtained for the 0.85 quantile (where 13 missed sequences corresponds to $1.69 \%$ and and the worst case corresponds to an $11.5 \%$ error) .

\section{CONCLUSIONS}

We used two fundamental results from the theory of extreme value distributions to select the GPDs as statistical model for the edge-like filter magnitudes over a high threshold. Removing the influence of the location parameter of the GPD (by shifting the threshold used to the origin) we obtain distributions in the two-parameter manifold of GPDs with fixed location parameter. For this manifold we computed the

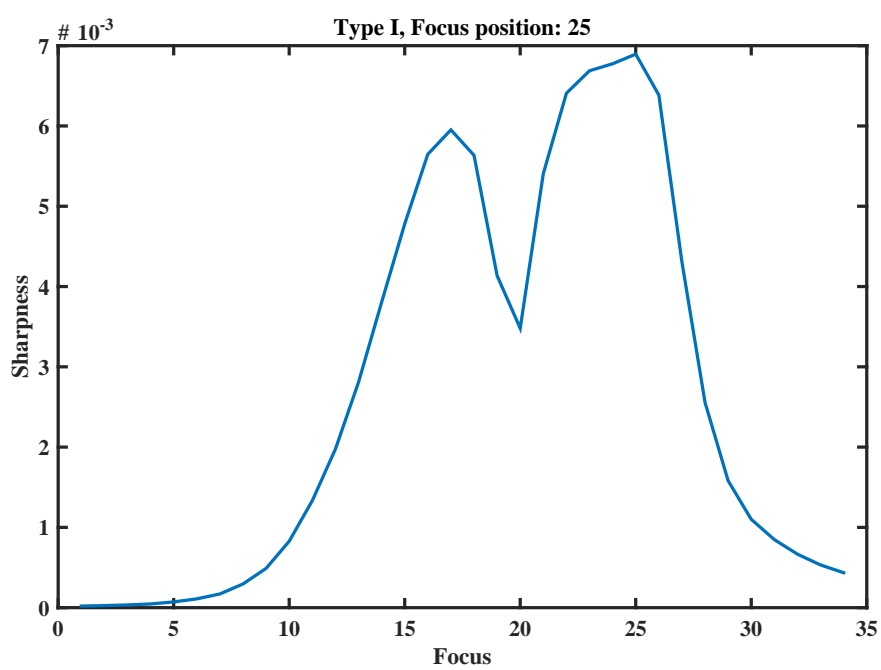

Fig. 10. Hoechst, focus position 25, sharpness function

Fisher information matrix describing the local geometry of this manifold. We then introduced the determinant of the inverse of the Fisher matrix as a sharpness function and showed that for a vast majority of sequences the autofocus process detected one of the slices in the ground truth focal region. The cases where it missed the correct region were either characterized by poor data quality or multiple maxima detection caused by contributions from neighboring slices. The results obtained show that GPD-based models provide enough information for the control of autofocus procedures. The form of the determinant clarifies also the role of the shape of the distribution and the variance in the determination of the focal plane. As a by-product we also gain additional computational 


\begin{tabular}{l|c|c|c|c|c} 
Quantile & 0.75 & 0.8 & 0.85 & 0.9 & 0.95 \\
\hline Hoechst, error & 40 & 34 & 28 & 31 & 38 \\
\hline Phalloidin, error & 18 & 15 & 13 & 25 & 89
\end{tabular}

TABLE I

Number of Focus Positions outside the Ground TRUth InTERVAL

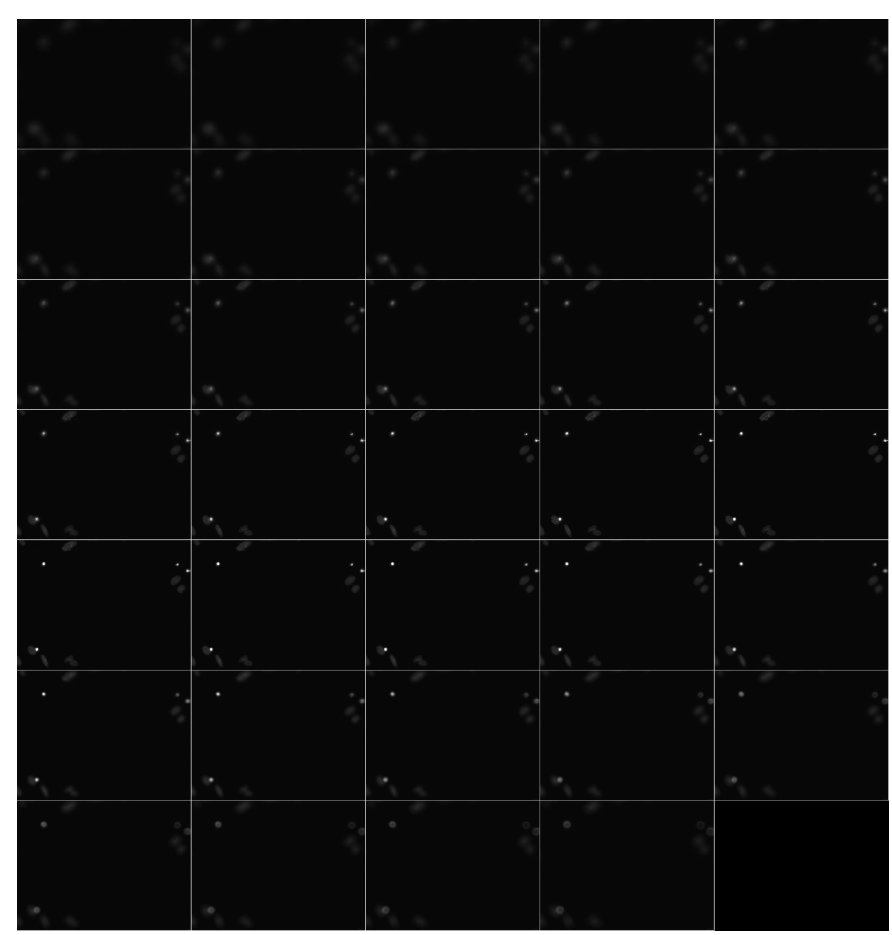

Fig. 11. Hoechst, focus position 25

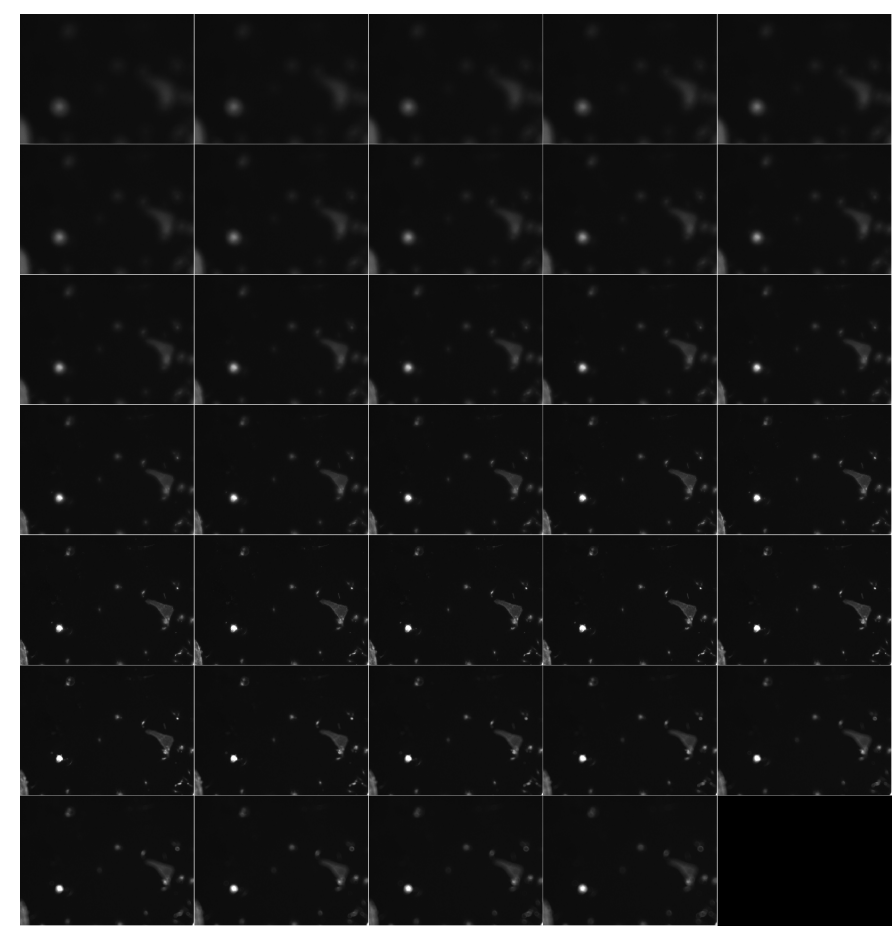

Fig. 12. Phalloidin, focus position 25

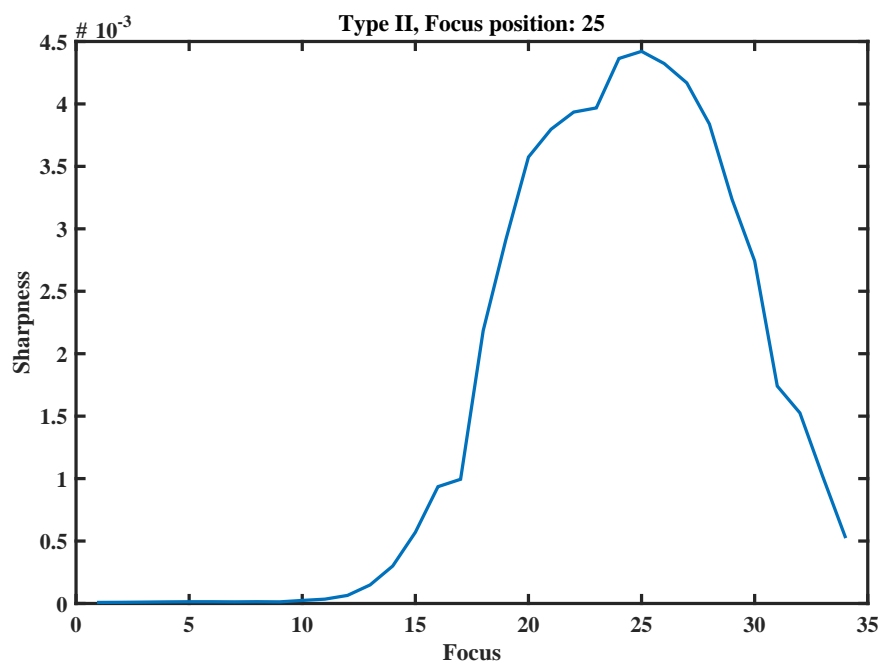

Fig. 13. Phalloidin, focus position 25, sharpness function

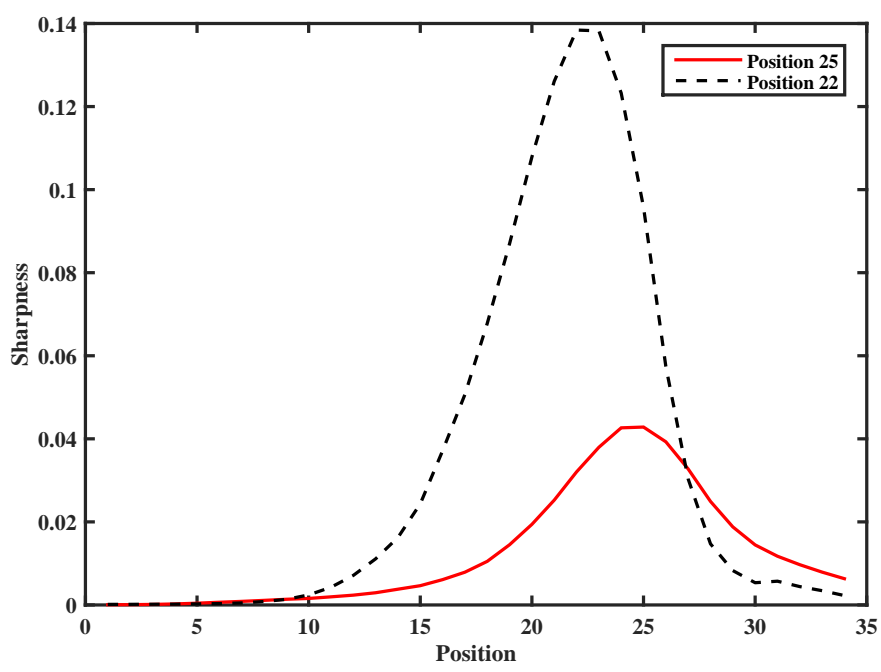

Fig. 14. Phalloidin, Focus positions 22 and 25, sharpness functions

efficiency since most of the pixels do not enter the sharpness computation. Another advantage of parametric models is the fact that they provide information about the analytical form of the sharpness function. This can be used in the construction of faster optimization methods where the sequence of an already measured set of parameters controls the step-length of the microscopes focus mechanism before the next image is collected [1]. We also want to point out that the autofocus method can also be used for other imaging devices since only the visual properties of the images enter the algorithm.

The computational bottleneck of the algorithm described so far is the distribution fitting which is an optimization problem that can be very time consuming when a large number of 
points enter the calculation. In a production implementation one should therefore avoid this step. One solution could be to avoid the distribution fitting all together. This could be achieved by starting from the assumption that the data follows a GPD. It is also easy to see that a strictly monotonic transformation of a sharpness function is a valid sharpness function. It should therefore be possible to modify the determinant based sharpness function so that it can be approximated by another function that depends only on variables that can be easily computed from the data. This new sharpness function would have the advantage that it is both, computationally efficient and based on verified statistical properties of the data.

\section{ACKNOWLEDGMENTS}

The support of the Swedish Research Council through a framework grant for the project Energy Minimization for Computational Cameras (2014-6227) and by the Swedish Foundation for Strategic Research through grant IIS11-0081 is gratefully acknowledged. We used the image set BBBC006v1 from the Broad Bioimage Benchmark Collection [4].

\section{REFERENCES}

[1] V. Zografos, R. Lenz, and M. Felsberg, "The Weibull manifold in lowlevel image processing: an application to automatic image focusing," Im. Vis. Comp., vol. 31, no. 5, pp. 401-417, 2013.

[2] R. Lenz, "Generalized extreme value distributions, information geometry and sharpness functions for microscopy images," in Proc. ICASSP, 2014, pp. 2867-2871.

[3] M. A. Bray, A. N. Fraser, T. P. Hasaka, and A. E. Carpenter, "Workflow and metrics for image quality control in large-scale high-content screens," J. Biomolecular Screening, vol. 17, no. 2, pp. 266-274, 2012.

[4] V. Ljosa, K. L. Sokolnicki, and A. E. Carpenter, "Annotated highthroughput microscopy image sets for validation," Nat Methods, vol. 9, no. 7 , p. $637,2012$.

[5] D.-C. Tsai and H. Chen, "Reciprocal focus profile," IEEE Transactions on Image Processing, vol. 21, no. 2, pp. 459-468, 2012.

[6] S. Pertuz, D. Puig, and M. A. Garcia, "Analysis of focus measure operators for shape-from-focus," Pattern Recognition, vol. 46, no. 5, pp. 1415 - 1432, 2013.

[7] R. Lenz, Group Theoretical Methods in Image Processing, ser. LNCS413. Springer, 1990

[8] — , "Investigation of receptive fields using representations of dihedral groups," J. Vis. Comm. Im. Repr., vol. 6, no. 3, pp. 209-227, 1995.

[9] H. S. Scholte, S. Ghebreab, L. Waldorp, A. W. M. Smeulders, and V. A. F. Lamme, "Brain responses strongly correlate with Weibull image statistics when processing natural images," J. Vision, vol. 9, no. 4, 2009.

[10] J.-M. Geusebroek and A. W. M. Smeulders, "Fragmentation in the vision of scenes." in Proc. ICCV, 2003, pp. 130-135.

[11] J.-M. Geusebroek, "The stochastic structure of images," in Scale Space, vol. LNCS-3459, 2005, pp. 327-338.

[12] Y. Jia and T. Darrell, "Heavy-tailed distances for gradient based image descriptors," in Adv. Neural Inf. Systems, 2011, pp. 1-9.

[13] V. Yanulevskaya and J.-M. Geusebroek, "Significance of the Weibull distribution and its sub-models in natural image statistics," in Proc. Int. Conf. Comp. Vision Theory and Appl., 2009, pp. 355-362.

[14] R. Fisher and L. Tippett, "Limiting forms of the frequency distribution of the largest or smallest member of a sample," Proc. Camb. Philos. Soc., vol. 24, pp. 180-190, 1928.

[15] B. Gnedenko, "Sur la distribuion limite du terme maximum d'une série aléatoire," Ann. Math., vol. 44, pp. 423-453, 1943.

[16] J. Pickands, "Statistical-inference using extreme order statistics," Ann. Statistics, vol. 3, no. 1, pp. 119-131, 1975.

[17] B. Finkenstadt and H. Rootzen, Extreme Values in Finance, Telecommunications, and the Environment. Taylor \& Francis, 2003.

[18] D. Clifton, L. Clifton, S. Hugueny, and L. Tarassenko, "Extending the generalised pareto distribution for novelty detection in high-dimensional spaces," J. Signal Processing Systems, vol. 74, no. 3, pp. 323-339, 2014.

[19] P. Embrechts, C. Klüppelberg, and T. Mikosch, Modelling extremal events : for insurance and finance. Berlin: Springer, 2003.
Reiner Lenz Reiner Lenz received his diploma degree in mathematics from the Georg August Universität in Göttingen, Germany and the $\mathrm{PhD}$. degree in Computer Engineering from Linköping University, Sweden. Before moving to Sweden he was a research assistant at Stuttgart University, Germany. His current research interests include group theoretical methods in signal processing, extreme value statistics and color signal processing. 